\title{
Comparison of Land Cover/Land Use and Habitat Classification Systems for Habitat Mapping from Space: Strengths and Weaknesses Evidenced in Mediterranean Sites of Natura 2000 Network
}

\author{
Valeria TOMASELLI ${ }^{1}$, Panayotis DIMOPOULOS ${ }^{2}$, Carmela MARANGI $^{3}$, \\ Athanasios S. KALLIMANIS ${ }^{2}$, Maria ADAMO ${ }^{4}$, Cristina TARANTINO ${ }^{4}$, \\ Maria PANITSA ${ }^{2}$, Massimo TERZI ${ }^{1}$, Giuseppe VERONICO ${ }^{1}$, \\ Francesco LOVERGINE ${ }^{4}$, Harini NAGENDRA ${ }^{5}$, Richard LUCAS $^{6}$, \\ Paola MAIROTA ${ }^{7}$, Caspar A. MUCHER ${ }^{8}$ and Palma BLONDA ${ }^{4}$ \\ ${ }^{1} \mathrm{CNR}-\mathrm{IGV}$, Bari/Italy · valeria.tomaselli@igv.cnr.it ${ }^{2}$ University of Ioannina/Greece \\ ${ }^{3} \mathrm{CNR}-\mathrm{IAC}$, Bari/Italy ${ }^{4} \mathrm{CNR}$-ISSIA, Bari/Italy \\ ${ }^{5}$ ATREE, Bangalore/India ${ }^{6}$ Aberystwyth University/UK \\ ${ }^{7}$ University of Bari/Italy ${ }^{8}$ ALTERRA, Wageningen/Netherlands
}

This contribution was double-blind reviewed as extended abstract.

\begin{abstract}
At a global level, protected sites have been established for the primary purpose of conserving biodiversity, with survey and monitoring of habitats undertaken largely within their boundaries. However, because of increasing human populations with greater access to resources, there is a need to now consider monitoring anthropic activities in the surrounding landscapes as pressures and disturbances are impacting on the functioning and biodiversity values of many protected sites. Earth Observation (EO) data acquired across a range of spatial and temporal scales offer new opportunities for monitoring biodiversity over varying time-scales, either through direct or indirect mapping of species or habitats. However, Land Cover (LC) and/or Land Use (LU), rather than habitat maps are generated in many national and international programs and, whilst the translation from one classification to the other is desirable, differences in definitions and criteria have so far limited the establishment of a unified approach. Focusing on both natural and non-natural environments associated with Natura 2000 sites in the Mediterranean, this paper considers the extent to which three common LC/LU taxonomies (CORINE, the Food and Agricultural Organisation (FAO) Land Cover Classification System (FAO-LCCS) and the IGBP) can be translated to habitat taxonomies with minimum use of additional environmental attributes and/or in situ data. A qualitative and quantitative analysis based on the Jaccard's index established the FAOLCCS as being the most useful taxonomy for harmonizing LC/LU maps with different legends and dealing with the complexity of habitat description and as a framework for translating EO-derived LC/LU to habitat categories. As demonstration, a habitat map of a wetland site is obtained through translation of the LCCS taxonomy.
\end{abstract}




\section{Introduction}

Earth Observation (EO) data and techniques are of great utility for assessing and monitoring biodiversity, not least because of the availability of a large amount of data from past, current and future-planned spaceborne missions. However, the focus on LC/LU mapping has detracted from the need to provide detailed information on habitats and, in many countries and regions, such mapping has already been put in place. Hence, in many cases, there is a need to translate these maps to those representing habitats with this undertaken through re-labelling and, where appropriate, merging of similar land cover classes (NAGENDRA 2001; LENGYEL et. al. 2008). Difficulties nevertheless arise because of different levels of definition and criteria used by specific classification systems. The FAO Land Cover Classification System (FAO-LCCS) has been identified as the most appropriate for providing a common language for translating and harmonizing different LC/LU legends. As habitat mapping is required, the majority of nations and regions have previously generated at least maps of land cover (LC) and land use (LU) using a range of schemes. The challenge, therefore, is to provide a mechanism for translating all schemes to a common taxonomy that also provides the best translation to a habitat taxonomy directly relevant to national and international reporting obligations. For this to be achieved, protocols are required to harmonize the difference systems and standardize new and pre-existing products for long-term monitoring purposes (HAINES YOUNG et al. 2000; DiMOPOULOS et al. 2005; BUNCE et al. 2008). The main purpose of this paper is to investigate the effectiveness of FAO-LCCS taxonomy to fill the gap between LC and habitat domains when mapping LC classes to habitats. A qualitative and quantitative analysis is carried out on Natura 2000 sites in Mediterranean countries (i.e., Italy and Greece).

\section{Analysis of Classification Systems in LC and Habitat Domains}

All the habitats characterizing the Mediterranean study sites have been listed and described according to different habitat classification schemes, i.e. Annex I, CORINE Biotopes, EUNIS and General Habitat Categories (GHCs), at the maximum available detail level. As well known, Annex I does not consider artificial and agricultural habitats, whose changes can affect the state of conservation of natural habitats. The Land Cover (LC) classes of the study sites have been described as both Corine Land Cover (CLC) and Land Cover Classification System (LCCS) classes. With regard to the usefulness of LCCS and CLC taxonomies for LC to habitat mapping, the main issues are highlighted hereafter: a) CLC description of vegetated classes corresponding to natural and semi-natural habitat types seems too vague; b) LCCS provides greater details than CLC. Very detailed LCCS classification levels can be reached by adding environmental and floristic attributes to "pure", land cover classifiers (DI GREGORIO \& JANSEN 2005). The use of LCCS environmental attributes, such as lithology, soils, landform and water quality improved the discrimination of LC classes up to the level of habitats. 


\section{A Quantitative Analysis}

In order to analyse the composition of LC/Habitat class types of the study sites in different LC/Habitat taxonomies, the Jaccard's Similarity Index was calculated for each pair wise comparison of all the sites. Given two sample sets, the Jaccard's index is calculated as the size (i.e. the cardinality) of the intersection divided by the size of the union of the two sets. Focusing on the classification systems, the highest values are the ones obtained by IGBP, due to the few and coarse classes that result in an artificial high number of overlaps. EUNIS, CORINE Biotopes and LCCS show low values on average, and also quite similar ranges. Regarding LCCS, as already stated, it is a very flexible tool, where class definitions can be enriched by adding further attributes to come to entities close enough. The detailed level of class description yields a better discrimination and a reduced number of cooccurrences in the site composition. The results of the similarity measure on the given dataset are then used to build up a distance matrix. About the comparison of Land Cover and Habitat taxonomies, the best results in the comparison are the ones obtained by LCCS with the addition of the environmental attributes which turns out to have the overall lowest distances to almost all habitat taxonomies but Annex I. The highest similarity is obtained for the coupling EUNIS - LCCS when the environmental attributes are taken into account.

\section{$4 \quad$ LC to Habitat Mapping}

A LC to habitat mapping application to IT4 site (Le Cesine) was carried out. Two preexisting maps at scale 1:5000 were available. The first map is a LC/LU map in CORINE taxonomy obtained by photo interpretation of an aerial image dated 2005 and validated by in-field campaigns carried out in 2007-2008. The second map is an habitat map realized during the same period by in-field campaigns, by integrating the ANNEX I with the EUNIS taxonomies.

We applied the LC to Habitat mapping to the CORINE and ANNEX I/EUNIS pre-existing maps of Le Cesine (IT4) in two steps. First the CORINE taxonomy of the original map was converted into LCCS taxonomy. Then, the conversion from LC to Annex I habitat was realized on the basis of habitat class description.

Decision Tables (DTs) were used to describe the complex relationships involved in the mapping process. The translation between LC/LU and habitat mapping was based on the additional thematic maps (i.e., lithology, soil surface aspect and soil subsurface aspect, water quality obtained by in-field campaigns) which, layered into a GIS, were used as inputs of the mapping process and the expert knowledge coded as decision rules in the Decision Table. Data from in-field campaign in this site were used as reference for validating the final habitat output map. A confusion matrix was generated to evaluate the mapping performance in terms of overall accuracy (OA) and error tolerance of the output map. The OA was obtained as the ratio of the number of patches in the output maps correctly assigned by the total number of patches considered. The resulting accuracy was $97 \%$ with an error tolerance of $0.02 \%$. 


\section{Conclusion and Outlook}

The qualitative comparison of CLC, IGBP and LCCS for a habitat mapping oriented applications in Mediterranean sites, indicates that LCCS allows a more precise land cover class definition of natural and semi-natural types by using the simple pure land cover classifiers. The use of LCCS environmental and technical attributes, whose selection depends on expert knowledge, appears mandatory for achieving an appropriate class descripttion very close to specific habitat types. The quantitative analysis based on similarity and congruency measurements carried out in this work demonstrates that LCCS shows the overall lowest distances (greatest similarity) to almost all habitat taxonomies but Annex I, with the greatest similarity in the case of EUNIS and GHC classification schemes, which share a similar building rule and do not only include vegetated habitats. To conclude, the coupling of LCCS taxonomy with GHCs and EUNIS classification schemes turns out to be the most appropriate for reliable long term biodiversity monitoring not only within Natura 2000 sites but also significantly in their surrounding areas as required for Biodiversity conservation policy definition. The BIO_SOS project (www.biosos.eu) has developed an accurate mapping between the two domain taxonomies (i.e. LC/ LU and habitats) based on their intrinsic definitions and classification rules as the initial step of the design and implementation of a biodiversity pre-operational monitoring system from space. In this work an application to the mapping LCCS to Annex I habitats is discussed and validated through available in-field data.

\section{References}

Bunce, R. G. H., Metzger, M. J., Jongman, R. H. G., Brandt, J., De Blust, G., ElenaRossello, R., Groom, G. B., Halada, L., Hofer, G., Howard, D. C., Kovàr, P., Mücher, C.A., Padoa Schioppa, E., Paelinx, D., Palo, A., Perez Soba, M., Ramos, I. L., Roche, P., SkÅnes, H. \& WrbKA, T. (2008), A standardized procedure for surveillance and monitoring European habitats and provision of spatial data. Landscape Ecol, 23, 11-25.

Di Gregorio, A. \& Jansen, L. J. M. (2005), Land Cover Classification System (LCCS): classification concepts and user manual. Food and Agriculture Organization of the United Nations, Rome.

Dimopoulos, P., Bergmeier, E. \& Fisher, P. (2005), Monitoring and conservation status assessment of habitat types in Greece: fundamentals and exemplary cases. Ann Bot, 5, 7-20.

HAINES-Young, R. \& WEBER, J. L. (2006), Land accounts for Europe 1990-2000. Towards integrated land and ecosystem accounting. EEA Report, 11. Copenhagen, European Environment Agency.

Lengyel, S., Kobler, S., Kutnar, L., Framstad, E., Henry, P.Y., Babi,, V., Gruber, B., SCHMELlER, D. \& HENLE, K. (2008), A review and a framework for the integration of biodiversity monitoring at the habitat level. Biodivers Conserv, 17, 3341-3356.

NAGENDRA, H. (2001), Using remote sensing to assess biodiversity. International Journal of Remote Sensing, 22, 2377-2400. 
